\title{
Back-n white neutron facility at CSNS and first-year nuclear data measurements
}

\author{
Jingyu Tang ${ }^{1,2,3}$ and the CSNS Back-n Collaboration \\ ${ }^{1}$ Institute of High Energy Physics, CAS, Beijing 100049, China \\ ${ }^{2}$ Spallation Neutron Science Center, Dongguan 523803, China \\ ${ }^{3}$ University of Chinese Academy of Sciences, Beijing 100049, China \\ Email: tangjy@ihep.ac.cn
}

\begin{abstract}
Back-streaming neutrons through the incoming proton channel at the spallation target station of China Spallation Neutron Source (CSNS) were exploited to build a white neutron beam line (the so-called Back-n). With a proton beam of $100 \mathrm{~kW}$ in beam power and $1.6 \mathrm{GeV}$ in kinetic energy and a thick tungsten target and modest moderation by the cooling water through the target slices, the neutron beam is very intense which is in the order of $7.0 \times 10^{6} \mathrm{n} / \mathrm{cm}^{2} / \mathrm{s}$ at $77 \mathrm{~m}$ from the target and has an excellent energy spectrum spanning from $0.5 \mathrm{eV}$ to $200 \mathrm{MeV}$. In addition, the time resolution related to the time-of-flight measurements is very good for neutron energy determination. Altogether, it makes the CSNS Back-n one of the best white neutron sources in the world and very suitable for nuclear data measurements, especially for neutron-induced cross-section measurements. Since the completion of the Back-n beamline and four physics spectrometers in March 2018, the first batches of experiments on nuclear data measurements have been carried out, which are summarized in this article.
\end{abstract}

\section{Introduction}

China Spallation Neutron Source (CSNS) is the largest scientific facility ever built in China, which was completed in March 2018 and serves mainly for multidisciplinary research on material characterization using neutron scattering techniques [1-3]. With the Phase-I (CSNS-I) goal of $100 \mathrm{~kW}$ in beam power, 1.6 $\mathrm{GeV}$ in beam energy and $25 \mathrm{~Hz}$ in pulse repetition rate, the CSNS accelerator complex is a powerful facility to support other research platforms besides neutron scattering, such as white neutron source, muon source and proton beams. For the future upgrading phase (CSNS-II), the beam power will be increased to $500 \mathrm{~kW}$.

The earlier studies show that the back-streaming neutrons along the impinging proton beam channel from the spallation target have properties of a wide energy spectrum, very high flux and good time structure, which is very suitable to be exploited as a white neutron source for nuclear data measurements and other applications [4-9]. With rapid development of nuclear energy in China, especially in advanced nuclear energy such as accelerator-driven subcritical systems (ADS), Thorium-based Molten Salt Reactor (TMSR) and fourth-generation reactors, very strong and eminent demands for much enhanced nuclear database have been increasing. There are also very high demands on specified nuclear data from nuclear astrophysics, fundamental nuclear physics and other research. As an extension of the CSNS engineering project during its construction phase, a project called the CSNS Back-n white neutron source was launched in 2013, and a consortium of five institutions was formed to support the design and construction of the beam line and spectrometers. To fully exploit the capability of the Back-n white neutron beam, a number of physics spectrometers dedicated to different types of measurements were planned, and four of them were made available for experiments in 2018. They are: four-unit C6D6 detectors [10] for neutron capture cross-section (n, $\gamma$ ) measurements, a multi-layer fast ionization chamber (FIXM) [11] for fission cross-section ( $n, f)$ measurements, a spectrometer (NTOX) for total cross-section (n, tot) measurements, a detector array (LPDA) for light-charged particle emission (n, lcp) measurement. An upgraded spectrometer based on $4 \pi \mathrm{BaF}_{2}$ array (GTAF-II) from the old version GTAF [12] will be ready for experiments by end 2019. Other planned spectrometers include: a new and larger $4 \pi \mathrm{BaF} 2$ array (GTAF-III) for neutron capture measurements, a detector array for prompt fission neutron spectrum measurements (PFNS), a $4 \pi \mathrm{HPGe} / \mathrm{LaBr}_{3}$ detector array for gamma spectrum measurements (n, $\mathrm{n}^{\prime} \gamma / 2 \mathrm{n} \gamma$ ), and a multi-function TPC (Time Projection Chamber) based spectrometer for measuring fission products and light charged particles. They are arranged in 
four locations in the two endstations, with the flight distances from the target of about $55 \mathrm{~m}$ and $76 \mathrm{~m}$, respectively, as shown in Fig. 1.

Though still at its early operation stage, the CSNS accelerator has been steadily increasing its beam power, from $10 \mathrm{~kW}$ in early 2018 to $50 \mathrm{~kW}$ in early 2019 . The first-year experiments on nuclear data measurements at the Back-n have been carried out very successfully.

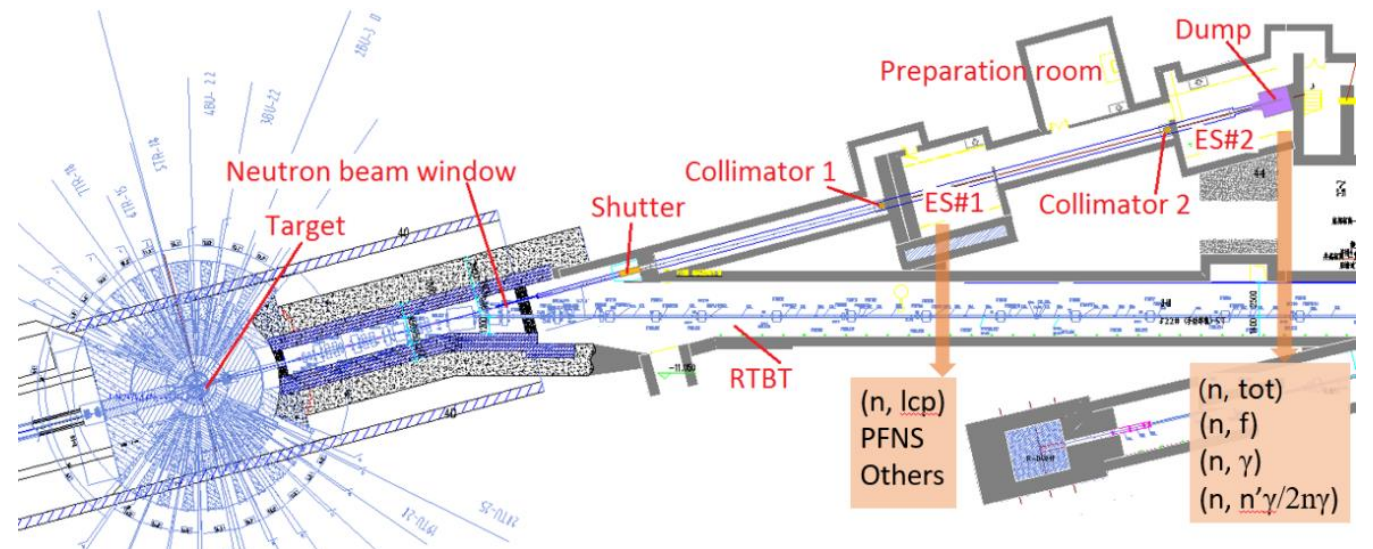

Fig. 1 Back-n white neutron beam line and spectrometers

Table 1. Different sets of beam spots, collimator apertures and neutron fluxes at Back-n at $100 \mathrm{~kW}$ in proton beam power. Except the first set which is for reducing fluxes, all the other aperture sets have been optimized according to the definition of the beam spot sizes at ES\#2.

\begin{tabular}{|c|c|c|c|c|c|c|}
\hline $\begin{array}{c}\text { Shutter } \\
(\mathrm{mm})\end{array}$ & $\begin{array}{c}\text { Coll\#1 } \\
(\mathrm{mm})\end{array}$ & $\begin{array}{c}\text { Coll\#2 } \\
(\mathrm{mm})\end{array}$ & $\begin{array}{c}\text { ES\#1 spot } \\
(\mathrm{mm})\end{array}$ & $\begin{array}{c}\text { ES\#1 flux } \\
\left(\mathrm{n} / \mathrm{cm}^{2} / \mathrm{s}\right)\end{array}$ & $\begin{array}{c}\text { ES\#2 spot } \\
(\mathrm{mm})\end{array}$ & $\begin{array}{c}\text { ES\#2 flux } \\
\left(\mathrm{n} / \mathrm{cm}^{2} / \mathrm{s}\right)\end{array}$ \\
\hline$\Phi 3$ & $\Phi 15$ & $\Phi 40$ & $\Phi 15$ & $1.3 \times 10^{5}$ & $\Phi 20$ & $4.6 \times 10^{4}$ \\
\hline$\Phi 12$ & $\Phi 15$ & $\Phi 40$ & $\Phi 20$ & $1.6 \times 10^{6}$ & $\Phi 30$ & $6.1 \times 10^{5}$ \\
\hline$\Phi 50$ & $\Phi 50$ & $\Phi 58$ & $\Phi 50$ & $1.6 \times 10^{7}$ & $\Phi 60$ & $6.9 \times 10^{6}$ \\
\hline $78 \times 62$ & $76 \times 76$ & $90 \times 90$ & $75 \times 50$ & $1.8 \times 10^{7}$ & $90 \times 90$ & $8.6 \times 10^{6}$ \\
\hline
\end{tabular}

\section{Back-n white neutron facility}

\subsection{White neutron beamline}

To exploit the back-streaming neutrons at CSNS, the proton beam line RTBT (Ring to Target Beam Transport) was modified to have a $15^{\circ}$ bending at a distance of about $20 \mathrm{~m}$ from the spallation target. The white neutron beam and the proton beam share the same beamline in this section of about $20 \mathrm{~m}$ in length. This bending magnet is also useful to remove the charged particles emitted from the target and the proton beam window. The influence of the proton beam window and two protective collimators in the common RTBT section to the neutron beam has been taken into account in the simulations.

A neutron beam window at the bending magnet is to separate the vacuums between the neutron beam line and RTBT. A thin Cadmium foil is optional to be inserted at the neutron window to absorb the neutrons with energy lower than $0.5 \mathrm{eV}$, in order to avoid the overlapping between different neutron pulses. Other types of absorbers can also be used for the in-beam background measurements. A neutron beam shutter just follows the neutron beam window, and with five positions and different apertures it can serve several functions: as the primary collimator, as the neutron stopper for the radiation safety of the endstations and as a valve to reduce neutron intensity. Two other collimators in the downstream together with the shutter are used to shape the beam profiles and adjust the intensities at the sample positions, as shown in Table 1 . The neutron fluxes with different beam spots at different locations have been confirmed by the measurements [13].

Careful shielding was applied to reduce both the neutron and gamma background in the two endstation halls (ES\#1 and ES\#2), and this includes the enforced shielding wall between the RTBT tunnel and ES\#1, the thick concrete walls after the shutter and the two collimators, a layer of Boron-containing polyethylene of $50 \mathrm{~mm}$ in thickness added to the ES\#1 wall, and a complicated in-house neutron dump. Both the simulations and measurements show that the ratio between the background and beam flux is from $10-5$ to $10-8$ for different aperture settings. For the absolute background without sample in beam, for example, it is about 0.5 $\mathrm{n} /\left(\mathrm{cm}^{2} . \mathrm{s}\right)$ and $0.5 \gamma /\left(\mathrm{cm}^{2} . \mathrm{s}\right)$ for off-beam background in ES\#1 for a beam spot of $\Phi 20 \mathrm{~mm}$, about $0.01 \mathrm{n} /\left(\mathrm{cm}^{2} . \mathrm{s}\right)$ and $0.01 \gamma /(\mathrm{cm} 2 . \mathrm{s})$ for off-beam background in ES\#2 for a beam spot of $\Phi 30 \mathrm{~mm}$. It is found that the scattered neutrons and gamma by the samples is overwhelmingly dominant in the endstations, which differ from one experiment to the other and should be solved carefully by the experiment people. The in-beam background from the 
scattered neutrons at the shutter, collimator, vacuum window, residual gas is still under investigation, both by simulations and measurements.

\subsection{Neutron energy spectrum}

Neutron beam characteristics are extremely important for cross-section measurements. Therefore, the production and moderation, and transport of neutrons in the tungsten target and along the beamline have been extensively simulated by using FLUKA [14-17] and benchmarked by MCNP. Detailed measurements for the neutron energy spectrum by using different methods were also carried, will be further pursued in the future.

In the simulations, the tungsten target size is taken as $6 \mathrm{~cm}(\mathrm{~V}) \times 16 \mathrm{~cm}(\mathrm{H}) \times 60 \mathrm{~cm}(\mathrm{~L})$, including the thin cooling-water layers [18]. The three moderators and the beryllium reflector above and below the target module, which are important for moderated neutrons for neutron scattering applications, are also included in the simulations. The footprint (four times the rms beam size) of the proton beam at the target entrance is $4 \mathrm{~cm}(\mathrm{~V}) \times 12$ $\mathrm{cm}(\mathrm{H})$, and the distribution is quasi-uniform [19]. Fig. 2 shows the neutron energy spectrum at Endstation\#2 where is about $80 \mathrm{~m}$ from the target, together with the measurements. There is a little difference in the spectrum shape for different collimator aperture settings and flight lengths. Two detectors were used for the measurements of the energy spectrum: a multi-layer ionization chamber with ${ }^{235} \mathrm{U}$ samples and an array of eight silicone detectors associated with a $\mathrm{LiF}$ sample foil. The former gives consistent results on the higher energy region with the simulations, while the latter on the lower energy range. One can see, with the Cadmium filter on, the neutron energy covers from $0.5 \mathrm{eV}$ to about two hundred $\mathrm{MeV}$ with a peak around $\mathrm{MeV}$. This wide energy range together with its very high flux and good time resolution provides an excellent beam not only for nuclear data measurements but also for other kinds of applications.

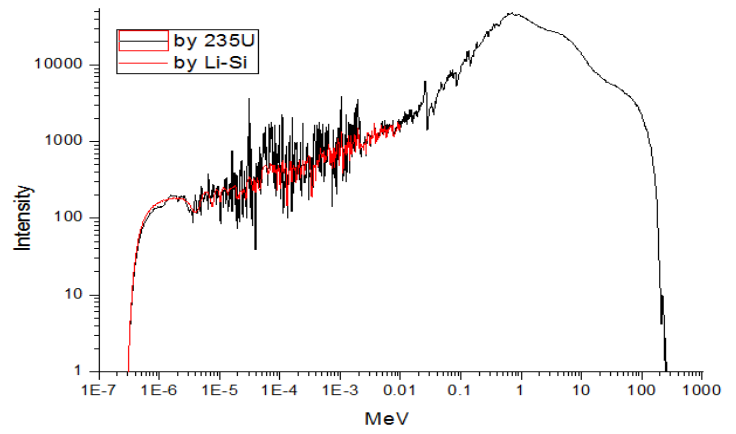

Fig.2. Simulated and measured neutron energy spectra at $76 \mathrm{~m}$ from the target (with the Cd filter on)

\subsection{Time-of-flight method}

The TOF (Time-of-Flight) method plays a pivotal role in determining neutron energy with a pulsed neutron source, and a good time resolution or energy resolution means a relatively long flight and a relatively short pulse length. For some experiments, very high time resolution is required to obtain the neutron energy determination of a few permille or even lower. At the CSNS Back-n, the flight length is limited to about $77 \mathrm{~m}$ and a thick target widens the neutron pulse width. Thus one can expect that the time resolution at Back-n should not be very high as compared to some white neutron beamlines with hundreds of meters at some facilities, and the simulations have confirmed this [6]. However, for fast neutrons, the time resolution is determined by the proton pulse width more than by the target thickness and moderation. One important issue here is that in the normal operation mode the proton pulses contain two bunches with an interval of 410 ns. This poses special difficulties for the measurements concerning the neutrons with energy higher than a few $\mathrm{keV}$. To solve this problem, two measures have been taken: one is to develop different dedicated operation modes for the CSNS accelerators to obtain short-bunch or single bunch proton beams [20]; the other is to develop the unfolding method to deconvolute the two bunches in one pulse. Both the single bunch mode and the unfolding method have been successfully applied to the experiment of the neutron energy spectrum measurement [21].

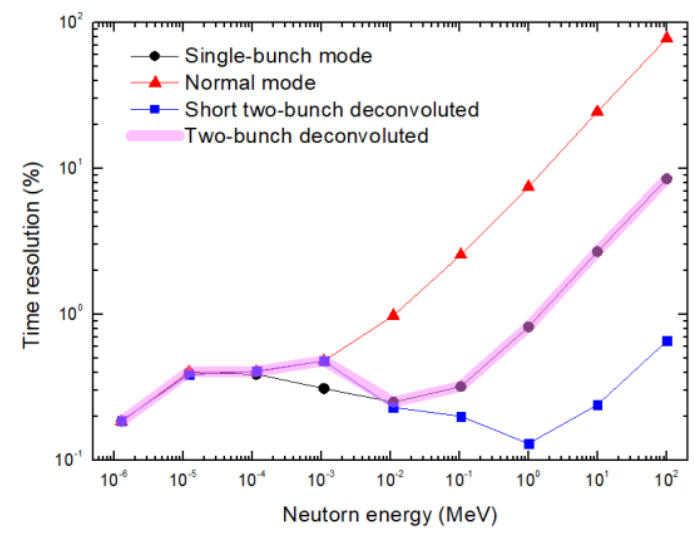

Fig.3 Time resolution with respect to neutron energy for the different operation modes (at $77 \mathrm{~m}$ from the target)

There are also errors in measuring the flight time of each detected neutron. One is from the so-called T0 signal that represents the neutron emission time from the target, and the other is from the arrival signal $\mathrm{T} 1$ at the detector. As it is not possible to measure the exact time of each neutron in a neutron pulse without losing it or changing its energy, we can only give the average starting signal in an indirect way, either from a proton bunch detection by a fast current monitor in the RTBT or by detecting the prompt gamma burst from the target in the neutron beam line. The gamma burst arrives at the detector earlier than the neutrons. Both methods are available at the Back-n. In most cases, the proton bunch signal is used in data acquisition after being calibrated with the gamma burst. As the Back-n is equipped with an advanced readout electronics system with waveform digitalization, some detectors which can detect both gammas and neutrons can use the gamma burst as the T0 signal directly. Otherwise, the gamma burst signal is provided from a dedicated plastic scintillator intercepting the gamma beam halo at the Collimator\#1. The T1 signal 
measurement depends on the detector type, with a precision varying from sub-nanosecond to a few nanoseconds. Fig. 3 shows the time resolution verse neutron energy for the different working modes. The time resolution in the level of a few per mille for the wide energy range at Back-n is considered well acceptable.

\subsection{Spectrometers for nuclear data}

\section{measurements}

As mentioned above, there are two endstation halls to host different spectrometers for nuclear data measurements and other experimental set-ups, with distances of about $55 \mathrm{~m}$ and $77 \mathrm{~m}$ from the spallation target, respectively. In total seven types of spectrometers have been planned: a 4-unit $\mathrm{C}_{6} \mathrm{D}_{6}$ detector and $4 \pi \mathrm{BaF}_{2}$ detector (GTAF-II or GTAF-III) array for neutron capture measurements, a multi-layer fission chamber (FIXM) for fission cross-section measurements, a combined detector system (NTOX) for total cross-section measurements, a 16-unit telescope array (LPDA) for light charged particle measurements, a fission PPAC plus 64-unit scintillator array (FINDA) for PFNS (Prompt Fission Neutron Spectrum) measurements, a combined $4 \pi \mathrm{HPGe} / \mathrm{LrBr}_{3}$ detector array for in-beam gamma spectrum measurements, and a multi-function TPC (Time Projection Chamber) for measuring fission products and light charged particles. Only five of them are in operation or available very soon, and introduced below in more details.

\subsection{1. $C_{6} D_{6}$ detectors}

This spectrometer [10] consists of four units of $\mathrm{C}_{6} \mathrm{D}_{6}$ detectors or hydrogen-free deuterated benzene liquid scintillators (Type: EJ315), which is similar to the one at CERN n_TOF [22-23], see Fig.4. The sample is in atmosphere, with a distance of about $150 \mathrm{~mm}$ to the detector centers. The supporting frame is made in aluminum with least material to reduce scattered gammas and neutrons. With a neutron capture reaction, there are usually several gamma emissions with different energies, but only one gamma is detected due to its small solid angle coverage. One also needs to introduce weighting functions in the data treatment to compensate the energy-dependent detection efficiency, and sometimes to correct the detection sensitivity of scattered neutrons. The spectrometer was among the first ones to carry out experiments at the Back-n from the beginning of 2018.

\subsubsection{FIXM}

FIXM (Fast Ionization Chamber Spectrometer for FIssion Cross Section Measurement) [11] is an eight-layer ionization chamber, as shown in Fig.5, which is similar to the one at CERN n_TOF [24]. A number of ${ }^{235} \mathrm{U}$ and ${ }^{238} \mathrm{U}$ samples are used as standard samples to calibrate neutron flux, and a maximum six experimental samples will take the rest sample positions. The structure design has been optimized to have a fast time response to obtain a better time resolution for neutron energy determination. The experiments with FIXM can cover a neutron energy range from $1 \mathrm{eV}$ to $20 \mathrm{MeV}$. The gamma burst can also be detected by FIXM and serves as the T0 signal. Although the relatively good detection efficiency with ${ }^{235} \mathrm{U}$ in low energy range, the rich resonances of ${ }^{235} \mathrm{U}$ pose some difficulties in obtain cross-sections with high precision, e.g. less than $3 \%$.

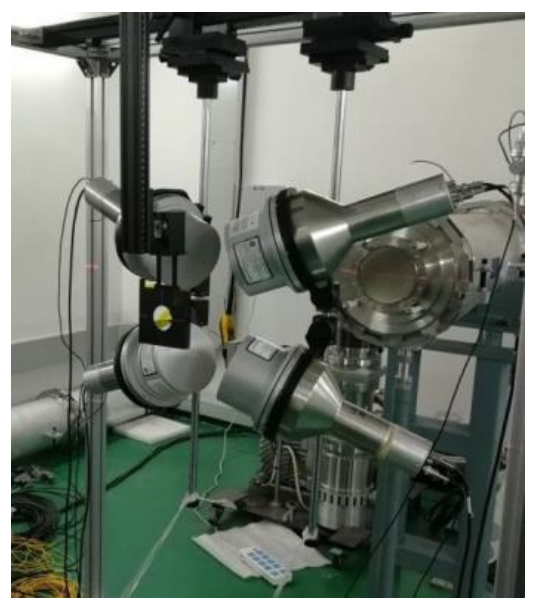

Fig.4. $\mathrm{C}_{6} \mathrm{D}_{6}$ detectors are for online experiment

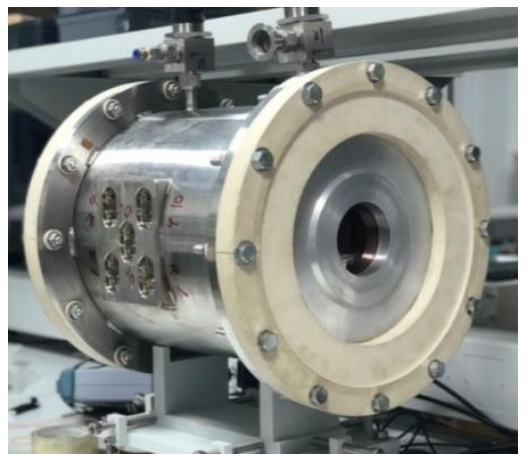

Fig.5. Schematic and photo for the FIXM spectrometer

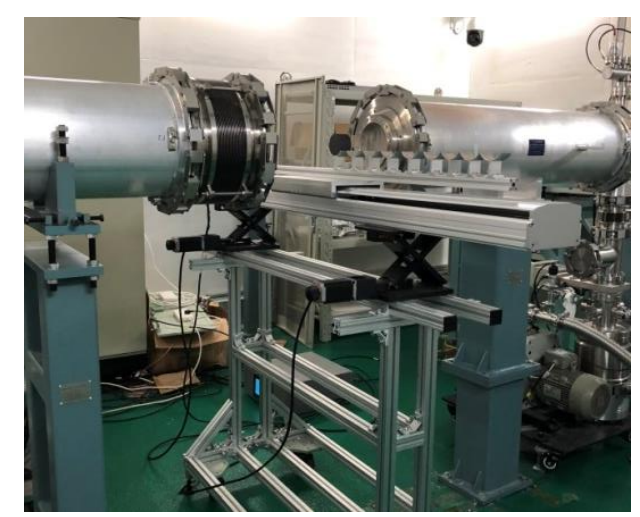

Fig. 6. Schematic for the NTOX spectrometer (upper) and sample changer (lower)

\subsubsection{NTOX}

NTOX (Neutron TOtal Cross Section Spectrometer) is composed of two major parts: a sample changing system 
located in the ES\#1 and a detector in the ES\#2, as shown in Fig. 6. It employs the transmission measurement method. The distance of about $20 \mathrm{~m}$ between the sample and the detector and the Collimator\#2 make sure that almost all the scattered neutrons will be cleaned in order not to produce background at the detector. Different detectors are planned to use for detecting neutrons in different energy range to assure high efficiency and high precision. In the last measurements, the FIXM was used, with ${ }^{235} \mathrm{U}$ and ${ }^{238} \mathrm{U}$ as standard samples. Other types of detectors such as scintillators are also under development.

\subsubsection{LPDA}

LPDA (Light-charged Particle Detector Array) is a spectrometer to be developed in steps. Sixteen detector units are planned for LPDA, and each detector unit has an MWPC-type gas chamber as low-energy $\Delta \mathrm{E}$ measurement, a silicon unit as higher-energy $\Delta \mathrm{E}$ measurement and a CsI(Tl) scintillator as the total energy measurement, as shown in Fig.7. This allows a large detection efficiency and large detection energy range of 0.5-100 MeV (proton) for light charged particles. A complex gas-flow control system allows precise pressure control for the vacuum chamber and the MWPC detectors. The rotatable detector support plate and sample changing systems make the experimental set-up flexible for each experiment, and one can even put a lead block here to absorb gammas for some experiments at ES\#2 or put the samples in vacuum for low-energy neutron total cross-section measurements. Although the whole system will be completed in late 2019 , the spectrometer has been employed for a number of experiments, mainly by using a 15-unit Silicone array, which will be presented in Section 3.

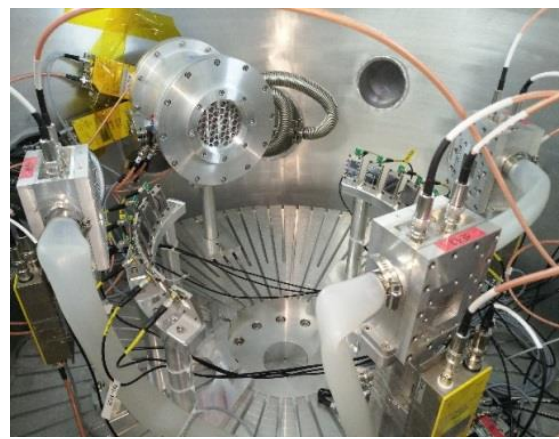

Fig.7. LPDA spectrometer under experiment

\subsubsection{GTAF-II}

GTAF-II is the upgraded version of GTAF (Gamma Total Absorption Facility) [12], which was constructed from 2003 at CIAE, initially for experiments at the HI-13 Tandem accelerator. It has 40 units of $\mathrm{BaF}_{2}$ detector ( 2 of 42 units leaving out for neutron beam pass) and covers about $90 \%$ of $4 \pi$ solid angle. With the upgrading, a totally new readout electronics which is more suitable to be with a high-intensity white neutron beam, a new mechanical support fitting the Back- $n$ condition and a neutron absorber shell in polyethylene plus Borium-10 are implemented, see Fig. 9. The on-site installation has been completed, and the first experiment at Back-n is planned to be in late 2019 .

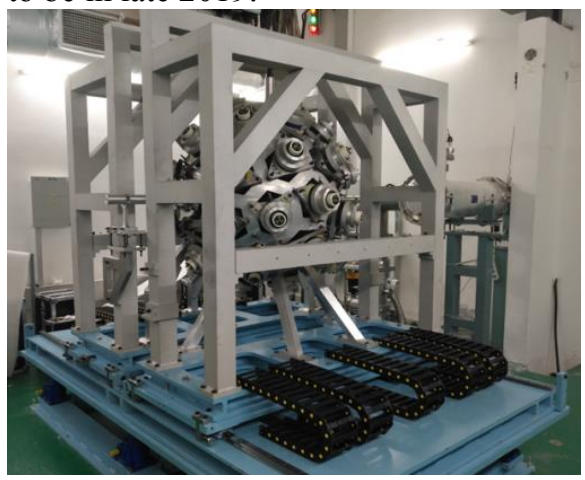

Fig.8. GTAF-II under installation at the Back-n ES\#2

\subsection{Generalized readout electronics and DAQ}

The generalized readout electronics, as shown in Fig. 10 , which is adaptable to any of the above spectrometers when it is online for experiments, is designed based on PXIe, wave-form digitization by folding-ADC and FPGA techniques, with a resolution of 12 bits and a sampling rate of 1 GSPS [25-26]. A digital trigger system that can be set according to different experiments is also used to reduce data volume to be recorded by DAQ. The system has been built up in steps, with 30 signal channels in operation since the end of 2018 , and 64 channels by the end of 2019, which can meet the requirements of all the detectors in service. As the large detector arrays will be put in service in a later phase, the actual DAQ is designed to receive data from the generalized electronics with transferring baud rate up to about $400 \mathrm{Mbps}$, and storing speed about $200 \mathrm{Mbps}$ after online software filter. The raw data is stored for limited period at the CSNS computation center thru 1 Gbps Ethernet. The DAQ has also expansibility to meet the future requirements.

\section{First-year nuclear data measurements}

After detailed measurements on beam characteristics and background, different kinds of experiments have started from April 2018, and most of them are nuclear data measurements. With the four available spectrometers that are for (n, $\gamma$ ) cross-sections, (n, f) cross-sections, (n, tot) cross-sections and (n, lcp) cross-sections, in total 19 different nuclei have been measured, as summarized in Table 2. This also includes one inelastic cross-section measurement $\left(n, n^{\prime} \gamma / 2 n \gamma\right)$ for ${ }^{56} \mathrm{Fe}$ by using two HPGe detectors. All these experiments demonstrate that the Back- $n$ is an excellent facility for nuclear data measurements based on the TOF method. Some experimental results have been written in papers which are either published or under review, and the others need more time for data analysis and paper writing.

\section{Conclusions}

As the first high-performance white neutron source in China, the CSNS Back-n white neutron source has been 
in operation since March 2018. The Back-n provides a neutron beam of very high flux, very wide energy spectrum from eV to $200 \mathrm{MeV}$ and good time structure, and is among the leading white neutron sources in the world, especially for its highest intensity. The neutron source serves wide applications and in particular nuclear data measurement. Among the eight types of spectrometers planned for nuclear data measurements, the four completed ones were employed in the experiments since March 2018. The fifth one will be ready by end 2019. In total, nineteen nuclei were measured for five different cross-sections in the first operation year, which show that very good quality data were obtained, though only a part of data was published.

Table 2. Nuclear data measurement experiments conducted at the Back-n

\begin{tabular}{|c|c|c|c|}
\hline Nucleus & Reaction & Spectrometer & Endstation \\
\hline${ }^{197} \mathrm{Au}$ & $(\mathrm{n}, \gamma)$ & $\mathrm{C}_{6} \mathrm{D}_{6}$ & $\mathrm{ES} \# 2$ \\
\hline${ }^{169} \mathrm{Tm}$ & $(\mathrm{n}, \gamma)$ & $\mathrm{C}_{6} \mathrm{D}_{6}$ & ES\#2 \\
\hline${ }^{57} \mathrm{Fe}$ & $(\mathrm{n}, \gamma)$ & $\mathrm{C}_{6} \mathrm{D}_{6}$ & ES\#2 \\
\hline${ }^{\text {nat }} \mathrm{Se}$ & $(\mathrm{n}, \gamma)$ & $\mathrm{C}_{6} \mathrm{D}_{6}$ & ES\#2 \\
\hline${ }^{89} \mathrm{Y}$ & $(\mathrm{n}, \gamma)$ & $\mathrm{C}_{6} \mathrm{D}_{6}$ & ES\#2 \\
\hline${ }^{\text {nat }} \mathrm{Er} /{ }^{162} \mathrm{Er}$ & $(\mathrm{n}, \gamma)$ & $\mathrm{C}_{6} \mathrm{D}_{6}$ & $\mathrm{ES} \# 2$ \\
\hline${ }^{232} \mathrm{Th}$ & $(\mathrm{n}, \gamma)$ & $\mathrm{C}_{6} \mathrm{D}_{6}$ & $\mathrm{ES} \# 2$ \\
\hline${ }^{238} \mathrm{U}$ & $(\mathrm{n}, \gamma)$ & $\mathrm{C}_{6} \mathrm{D}_{6}$ & $\mathrm{ES} \# 2$ \\
\hline${ }^{93} \mathrm{Nb}$ & $(\mathrm{n}, \gamma)$ & $\mathrm{C}_{6} \mathrm{D}_{6}$ & ES\#2 \\
\hline${ }^{12} \mathrm{C}$ & (n, tot) & NTOX & $\mathrm{ES} \# 1+2$ \\
\hline${ }^{27} \mathrm{Al}$ & (n, tot) & NTOX & $\mathrm{ES} \# 1+2$ \\
\hline${ }^{235} \mathrm{U}$ & $(n, f)$ & FIXM & ES\#2 \\
\hline${ }^{238} \mathrm{U}$ & $(n, f)$ & FIXM & ES\#2 \\
\hline${ }^{236} \mathrm{U}$ & $(n, f)$ & FIXM & ES\#2 \\
\hline${ }^{232} \mathrm{Th}$ & $(\mathrm{n}, \mathrm{f})$ & FIXM & $\mathrm{ES} \# 2$ \\
\hline${ }^{6} \mathrm{Li}$ & (n, lcp) & LPDA & ES\#1 \\
\hline${ }^{10} \mathrm{~B}$ & (n, lcp) & LPDA & ES\#1 \\
\hline${ }^{1} \mathrm{H}$ & $(\mathrm{n}, \mathrm{lcp})$ & LPDA & ES\#1 \\
\hline${ }^{56} \mathrm{Fe}$ & $\left(\mathrm{n}, \mathrm{n}^{\prime} \gamma\right)$ & 2-unit HPGe & ES\#2 \\
\hline
\end{tabular}

\section{Acknowledgements}

This work is jointly supported by the National Key Research and Development Program of China (Project: 2016YFA0401600), National Natural Science Foundation of China (Project: 11235012), the CSNS Engineering Project, and the Back-n Collaboration Consortium fund. The authors would like to thank the colleagues from CSNS and the five supporting institutions for all kinds of support.

\section{References}

1. S. X. Fang et al., J. Kor. Phys. Soc., 48 (2006) 697.
2. J. Wei, H.S. Chen, Y.W. Chen et al., Nucl. Instr. and Meth., A600 (2009) 10-13.

3. Hesheng Chen and Xun-Li Wang, Nature Materials 15, (2016) 689-691.

4. J. Y. Tang, S.N. Fu and J. Wei, J. Kor. Phys. Soc., 52(2008) 710 .

5. J.Y. Tang, S.N. Fu, H.T. Jing, et al., Chinese Physics C, 2010, Vol. 34 (01): 121-125

6. H.T. Jing, J.Y. Tang, H.Q. Tang, et al., Instr. and Meth., A 621 (2010) 91-96

7. TANG Jing-yu, JING Han-tao, XIA Hai-hong, et al., Atom. Ener. Sci. and Tech., Vol. 47, No.7 (2013) 1089-1095

8. JING Han-Tao, TANG Jing-Yu, YANG Zheng, Chinese Physics C, Vol. 37, No. 11 (2013) 117002

9. Q. An, H.Y. Bai, J. Bao et al. (the CSNS Back-n collaboration), JINST 12 (2017) P07022

10. J. Ren, X. Ruan, J. Bao, et al. Radiat Detect Technol Methods (2019) 3: 52.

11. Yiwei Yang a, Zhongwei Wen, Zijie Han et al., Nucl. Instr. and Meth., A 940 (2019) 486-491

12. J. Zhao, G. He, Y. Yan, et al. Atomic Energy Science and Technology, 47(4), (2013) 669-673 (in Chinese)

13. Bao Jie, Chen Yong-Hao, Zhang Xian-Peng et al., Acta Phys. Sin. Vol. 68, No. 8 (2019) 080101 (in Chinese)

14. G. Battistoni, S. Muraro, P.R. Sala, et al., AIP Conf. Proc. 896 (2007) 31-49.

15. A. Ferrari, P.R. Sala, A. Fassò, and J. Ranft, CERN 2005-10,,INFN/TC-05/11, SLAC-R-773, 2005.

16. L.Y. Zhang, H.T. Jing, J.Y. Tang, X.Q. Wang, Rad. Phys. and Chem. 127 (2016) 133-139

17. L.Y. Zhang, H.T. Jing, J.Y. Tang, et al. Applied Radiation and Isotopes. 132 (2018) 212-221

18. FangWei Wang, TianJiao Liang, Wen Yin, et al., Science China Phys., Mech. and Astr., Vol. 56, (2013) 2410-2424

19. J. Y. Tang, G. H. Wei and C. Zhang, Nucl. Inst. and Meth. in Phys. Res., A582 (2007) 326-335

20. Ye Zou, Jingyu Tang, Jinfang Chen, et al., Phys. Rev. ST Accel. Beams 17, 060101 (2014)

21. Yonghao Chen, Guangyuan Luan, et al., Eur. Phys. J. A (2019) 55: 115

22. R. Plag, M. Heil, F. Käppeler et al., Nucl. Instr. and Meth. A496 (2003) 425

23. F. Gunsing et al., The n_TOF collaboration, Eur. Phys. J. Plus (2016) 131:37̄1

24. M. Calviani, P. Cennini, D. Karadimos, et al. Nucl. Instr. and Meth. A 594, (2008) 220

25. Deliang Zhang, Ping Cao, Qi Wang, et al., Chinese Physics C, Vol. 41, No. 2 (2017) 026102

26. T. Yu, P. Cao, X.Y. Ji, et al., IEEE Trans. Nucl. Sci. Vol. 66, No. 7 (2019) 1095-1099 